\title{
Characteristics of Peripheral Blood Cells in COVID- 19 Patients Revealed by a Retrospective Cohort Study
}

\section{Xunliang Tong}

Beijing Hospital

\section{Anqi Cheng}

China-Japan Friendship Hospital

\section{Xueting Yuan}

Beijing Hospital

\section{Xuefeng Zhong}

Beijing Hospital

\section{He Wang}

Beijing Hospital

\section{Wei Zhou}

Beijing Hospital

Xiaomao Xu

Beijing Hospital

Yanming Li ( $\square$ lymyl@263.net )

Department of Pulmonary and Critical Care Medicine, Beijing Hospital, National Center of Gerontology; Institute of Geriatric Medicine, Chinese Academy of Medical Sciences, P. R. China;

\section{Research}

Keywords: COVID-19, lymphocytes, eosinophils, prognosis, immune response

Posted Date: October 22nd, 2020

DOI: https://doi.org/10.21203/rs.3.rs-94972/v1

License: (c) (i) This work is licensed under a Creative Commons Attribution 4.0 International License. Read Full License 


\section{Abstract}

\section{Background}

Peripheral hematological changes in severe COVID-19 patients may reflect the immune reaction during SARS-CoV-2 infection. Characteristics of peripheral blood cells as early signals were needed to be investigated for clarifying its associations with the fatal outcomes in COVID-19 patients.

\section{Methods}

A retrospective cohort study was performed and the hospitalized COVID-19 patients were recruited in wards of Tongji Hospital (Wuhan, China). Characteristics of peripheral blood cells in survivors and nonsurvivors were analyzed. Also the comparison among patients with different level of eosinophils was performed.

\section{Results}

198 patients were included in this study, of whom 185 were discharged and 13 died in hospital. Compared to the survivors, counts of lymphocytes, monocytes, eosinophils and basophils were significantly decreased in non-survivors. According to the level of eosinophils, patients were divided into low EOS group $\left(<0.02 \times 10^{9} / \mathrm{L}\right)$ and normal EOS group $\left(\geq 0.02 \times 10^{9} / \mathrm{L}\right)$. Patients in the low EOS group showed a significantly higher fever compared to normal EOS group. The proportion of patients in low EOS group who used glucocorticoids increased significantly than the other group. Death rate in the low EOS group was higher and no patient death in normal EOS group. Moreover, positive correlation was found between the counts of lymphocytes and eosinophils in patients with glucocorticoids use but not in patients without the treatment.

\section{Conclusions}

Hematological changes differed between survivors and non-survivors with COVID-19. Lymphopenia and eosinopenia could serve to predict the poor prognosis of COVID-19 patients. Initial counts of eosinophils may guide us in usage of glucocorticoids for COVID-19 treatment.

\section{Background}

The outbreak of COVID-19 which caused by the infection of severe acute respiratory syndrome coronavirus 2 (SARS-CoV-2) has rapidly spread throughout the world [1, 2]. The clinical manifestation of COVID-19 could specifically display in a wide spectrum, which is so far mostly mild and self-limiting. Besides, other COVID-19 patients show severe viral pneumonia with respiratory dysfunction, even including several organs failure, resulting in $2 \%$ to $3 \%$ mortality rate in the worldwide [3].

With advanced knowledge of human coronary virus, the immune dysfunction was observed in COVID-19 patients. In mild cases, immune responses were efficiently established to curb the viral replication [4]. 
However, in severe cases, uncontrolled inflammation and the microcirculation dysfunctions together lead to viral sepsis with immunologic impairment [4]. The severity of disease was associated with immunological impairment. Especially, in some life-threaten cases, SARS-CoV-2 could trigger catastrophic damage to the human immune system resulting in death at their worst.

Unfortunately, our understanding of immune response to SARS-CoV-2 is extremely limited until now. Many scholars speculated that the interaction of SARS-CoV-2 and host could be referring to the other coronavirus because of the highly similarity in the sequence homology in coronavirus family [5]. Previous study mainly focused on the immune dysfunction caused by severe acute respiratory syndrome coronavirus (SARS-CoV) and middle east respiratory syndrome coronavirus (MERS-CoV), respectively. Coronavirus infections (SARS and MERS) are confirmed to activate both innate and adaptive immune responses, which included that lymphopenia and thrombocytopenia caused by SARS-CoV due to immune complexes and T cells' apoptosis through extrinsic and intrinsic pathways triggered by MERS-CoV $[6,7]$. In simply means that the changes of peripheral blood cells could reflect the immune damage caused by virus infection.

Lymphocytes play a crucial role in maintaining immune homeostasis during virus infection, especially SARS-CoV-2[8]. Several cohort studies have reported lymphopenia can predict prognosis in COVID-19 patients $[9,10]$. In addition, a few studies found that the eosinopenia was associated with poor prognostic features. Thus, the differentiation of peripheral blood cells may indicate the immunologic impairment at the early stage of the disease. However, the risk factors for the changes of peripheral blood cells in the prognosis are not well addressed yet. A retrospective cohort study predicted the value of peripheral blood cells in COVID-19 patients was performed for further investigation.

\section{Methods}

\section{Study Design}

As one-time supportive task led by China National Health Commission, patients diagnosed of COVID-19 according to World Health Organization interim guidance were under treatment by medical team from Beijing Hospital at designated wards in the Sino-French New City Branch of Tongji Hospital in Wuhan, Hubei province, China. After excluding patients with hematological disease or a blood transfusion in a week following admission, a total of 198 cases with the clarified outcome (discharged or deceased) were included by the mission complete. A retrospective cohort study was performed. The severity of the disease was assessed according to the Seventh Version of the Novel Coronavirus Pneumonia Diagnosis and Treatment Guidance from the National Health Commission of China. The study was approved by Ethics Committee of Beijing Hospital (2020BJYYEC-046-01)

\section{Medical data extraction}

The clinical data, including demographics information; clinical symptoms and signs; underlying diseases; laboratory results; the most intense level of oxygen support; treatment and clinical outcomes, were 
extracted from electronic medical records. The whole laboratory evaluation was consisted of complete blood cell counts, biochemical and coagulation indices and so forth. The differential peripheral blood indices were detailed recorded. The baseline data were recorded in the first 24 hours after admission. The end point was written of discharging from hospital or death. The differences in clinical characteristics and laboratory findings in patients with different outcome would be addressed. Longitudinal tracing of laboratory indices during the hospitalization was performed. Furthermore, independent predicting factors associated with the fatal outcome would also be investigated. All the data were entered into a computerized database and checked by two experienced physicians independently.

\section{Statistical analysis}

Continuous variables were described using median and interquartile range (IQR). Categorical variables were described as number (\%). Non-normal distributed continuous data were compared using MannWhitney-Wilcoxon test. Categorical data were compared using $X^{2}$ test or the Fisher exact test. Correlations between variables were analyzed using the Spearman's rank correlation. Correlation strength was selected by an absolute correlation $|r|>0.2$ and the selected correlation were plotted as an undirected network graph. All tests were 2 -sides, and a $P$ value $<0.05$ was considered statistically significant. Data was analyzed using IBM SPSS Statistics software (version 19.0).

\section{Results}

\section{Demographic information of survivors and non-survivors}

A total of 198 patients confirmed severe COVID-19 were enrolled in this study. The median age of patients and the gender distribution between two groups (survivor group and non-survivor group) was basically the same. Majority of the included patients in both two groups were with comorbidity and more than half of the patients had at least one underlying disease. The ranking of the underlying disease was hypertension (40.0\%), diabetes (16.7\%), chronic respiratory disease $(5.7 \%)$, cardiovascular disease $(5.2 \%)$ and so on. Among the underlying disease, the percentage of malignant disease of patients in the nonsurvivor group is higher than it in the other group. The most common symptoms on admission were fever and cough, followed by fatigue and sputum production in both two groups. During the clinical treatment, the most intense level of oxygen support was recorded. Patients in survivor group were mostly under oxygen therapy by nasal cannula in survivors compared to patients in non-survivor group (47\% vs $0, P$ $<0.05$ ). The proportion of patients under invasive ventilation (IMV) in non-survivors was significantly higher than that in the other group ( $53.8 \%$ vs $2.2 \%, P<0.05)$. More than $70 \%$ of the patients received antivirals, and Lopinavir/Ritonavir use differed significantly between non-survivors and survivors ( $6.7 \%$ vs $100.0 \%, P<0.05)$. According to the CURB-65 score, the proportion of patients with different grade showed significant differences between two groups. Systematic glucocorticoids use differed significantly between non-survivors and survivors $(66.7 \%$ vs $22.5 \%, P=0.002)$. At the end of the observing period, 185 (93.4\%) patients had been discharged and 13 (6.6\%) patients had died. Demographic information showed in Table 1. 


\section{Initial laboratory findings among survivors and non-survivors}

Hematologic profile on admission varied among patients which hinted with different outcomes. Lymphocytes (LYM), monocytes (MONO) and platelets (PLT) were dramatically decreased in nonsurvivors with significant differences compared to the survivors (LYM: $10.9 \%$ vs $24.5 \%, P<0.05$; MONO: $5.9 \%$ vs $8.7 \%, P<0.05 ; \mathrm{PLT}: 145[76,241] \times 10^{9} / \mathrm{L}$ vs $\left.232[171,305] \times 10^{9} / \mathrm{L}, P<0.05\right)$. Moreover, compared to survivors, the counts of eosinophils (EOS) and basophils (BASO) in non-survivors were too low to be detected and to analysis. In contrast with survivors, level of neutrophils (NEU) and the ratio of neutrophilsto-lymphocytes ratio (NLR) were significantly elevated in non-survivors (NEU: $83.6 \%$ vs $65.5 \%, P<0.05$; NLR: 8.43 vs $3.05, P<0.05)$. The percentage alteration was in consistent with the absolute counts change for each analyzed peripheral blood cells. Moreover, compared with survivors, levels of C-reactive protein (CRP), IL-6, and serum ferritin were significantly increased in non-survivors with statistical differences. Regarding the coagulation parameters, the prolonging of PT and increased levels of D-dimer were significantly higher in non-survivors compared to survivors (Table 2; Figure 1).

\section{Effects on clinical characteristics of different EOS levels in COVID-19 patients}

According to the level of circulating EOS counts, COVID-19 patients were divided into two groups: low EOS group $\left(<0.02 \times 10^{9} / \mathrm{L}\right)$ and normal EOS group $\left(\geq 0.02 \times 10^{9} / \mathrm{L}\right)$. The media age and gender distribution between the two groups without significant differences (age: 64[53, 71] yrs vs 61[47, 69] yrs, $P=0.126$; gender (male): $52.6 \%$ vs $48.3 \%, P=0.561$ ). Fever of patients in the low EOS group were higher compared with normal EOS group with significant differences, rather than other symptoms $\left(38.9[38.4,39.0]^{\circ} \mathrm{C}\right.$ vs $\left.38.5[38.0,39.0]{ }^{\circ} \mathrm{C}, P=0.011\right)$. The severity of disease was evaluated by CURB-65 score. The percentage of CURB-65 score of patients between the two groups was differed with significant differences. The proportion of patients in low EOS group who used glucocorticoids was significantly higher than that of normal EOS group ( $44.8 \%$ vs $12.5 \%, P<0.05)$. Duration of viral shedding in two groups showed no significant difference. The time from illness onset to discharge in low EOS group significantly longer than the other group $(28[23,35] \mathrm{d}$ vs $25[20,32] \mathrm{d}, P=0.046)$. Moreover, death rate in the low EOS group was significantly higher and no patient death in normal EOS group ( $16.7 \%$ vs $0, P<0.05)$ (Table 3$)$.

\section{Correlation networks analysis for peripheral blood cells}

On admission, we observed a positive correlation between the counts of NEU and MONO ( $r=0.549)$, NEU and PLT $(r=0.530)$ in non-survivors, but not in survivors. When at end-hospitalization, strong correlations between counts of NEU and MONO ( $r=0.771)$, NEU and EOS $(r=0.735)$, NEU and BASO $(r=0.623)$ were observed in non-survivors. Furthermore, non-survivors showed similar positive strong correlations between the counts of EOS and BASO $(r=0.562)$ (Figure 2).

Patients who received glucocorticoids therapy showed a negative correlation between the counts of WBC and LYM $(r=-0.265)$, but a positive correlation between WBC and LYM was observed in patients without glucocorticoids therapy $(r=0.531)$. After glucocorticoids treatment, the counts of EOS negatively correlated with NEU $(r=-0.288)$, but no correlation was observed before the treatment $(r=0.058)$. A 
similar correlation was observed between the counts of LYM and EOS in patients received glucocorticoids therapy $(r=0.454)$, but no correlation was found before the treatment $(r=0.020)$ (Figure 3).

\section{Discussion}

As the classification of survivors and non-survivors was observed in this cohort study, the differential features of peripheral hematologic cells were analyzed. Previous study demonstrated that severe patients tend to have lower lymphocytes counts, higher leukocytes counts and neutrophil-lymphocyte-ratio (NLR), as well as lymphopenia has been reported as a predictor of prognosis in COVID-19 patients $[9,11,12]$. Our data also revealed that the initial counts of lymphocytes, eosinophils, and basophils of COVID-19 patients were much more decreased in non-survivors compared with the counts of above indices in survivors, which with the consistent conclusion with other studies.

Eosinophils are linked to immune response conferring host protection against viruses and eosinopenia has been observed in different acute inflammation situation as pneumonia [13-15]. A recent report by Xie et al proved that COVID-19 patients with low EOS counts were likely to have more severe symptoms such as fever, fatigue, shortness of breath, more lesions in chest CT, radiographic aggravation, longer length of hospital stay and course of disease [16]. Our study also indicated that patients with low EOS on admission showed a significantly higher fever. Eosinopenia may be the result of rapid sequestration of circulating eosinophils mediated by the overwhelming release of inflammatory cytokines, including thermogenic ones (such as IL-1, IL-6) [17]. In addition, effects of glucocorticoids on hematological and immunological indicators were significant especially the decrease in counts of eosinophils.

Although our understanding of the specific innate and adaptive immune response to SARS-CoV-2 is relatively limited, the hematological changes may reflect a homeostatic mechanism to prevent systemic over-activation of inflammation. SARS-CoV-2 RNA and proteins interact with various pattern recognition receptors can initiate antiviral immune responses which characterized by differentiation and proliferation of various immune cells with immune mediator production and release, especially lymphocytopenia and elevated level of IL-1 $\beta$, IFN-y, IP-10 and IL-17, regulating viral replication and spreading within the host [18, 19]. SARS-CoV-2 has been proven to induce remodeling of peripheral lymphocytes, and a more robust humoral immune response occurs in severe infection [20]. The decreased production, apoptosis and redistribution of lymphocytes may together lead to circulating lymphopenia [21]. In addition, eosinophils are recruited from the blood circulation into the inflammatory focus, modulating immune responses through releasing a serious of cytokines and other mediators, as well as by a broad spectrum of immune mechanisms [22]. In short, uncontrolled SARS-CoV-2 infection and the immune response may cause a systemic destruction, while the changes of peripheral blood cells can serve as early signals of immune impairment in COVID-19 patients [23].

Glucocorticoids can avoid excessive inflammation by inhibiting immune response to SARS-Cov-2 infection, while the suppression of immunity may lead to an increase in viral load [24]. Besides, glucocorticoids can suppress the release of EOS in bone marrow and promote eosinophil clearance by 
directly inducing apoptosis $[25,26]$. The panel of WHO made a strong recommendation for use of glucocorticoids in severe and critical COVID-19 patients, and in a real-life clinical setting, physicians tend to use glucocorticoids in most critically patients [27]. In this cohort, we proved that the use of glucocorticoids altered the immunological characteristics of peripheral blood cells and glucocorticoidrelated EOS decreased, which was considered as a risk factor for fatal outcomes. Due to the role of glucocorticoids in treating severe COVID-19 patients is still controversial, blood immunological marker which could be used as an index to guide the strategy of glucocorticoids therapy in COVID-19 patients is needed and may improve the prognosis in the clinical practice.

Early identification of risk factors for critical illness can facilitate appropriate provision of supportive care and help reduce mortality. Blood routine seems like a convenient and effective indicator which can help to identify the entities involved in immune dysregulation. Lymphopenia and eosinopenia on admission may be particularly important to indicate the poor prognosis of COVID-19 patients, and counts of eosinophils are of guiding significance for the use of glucocorticoids. In conclusion, peripheral blood cells may serve as early signals of disease progression, which can be chosen as effective monitor parameters during the treatment of COVID-19.

\section{Abbreviations}

APTT: activated partial thromboplastin time; BASO :basophils; CRP :C-reactive protein; COVID-19: coronavirus disease 2019; EOS: eosinophils; FIB: fibrinogen; IQR: interquartile range; LDH: lactate dehydrogenase; LYM: lymphocytes; MV: mechanical ventilation; MERS-CoV: middle east respiratory syndrome; MONO: monocytes; NMV: noninvasive methods of mechanical ventilation; NT-proBNP: Nterminal pro brain natriuretic peptide; NEU: neutrophils; PT: Prothrombin time ; RT-PCR: real-time reverse transcriptase polymerase-chain reaction; SARS-CoV: severe acute respiratory syndrome; SARS-CoV-2: severe acute respiratory syndrome coronavirus; WBC: white blood cell; WHO: World Health Organization;

\section{Declarations}

\section{Ethics approval and consent to participate}

The study was approved by Ethics Committee of Beijing Hospital (2020BJYYEC-046-01).

\section{Consent for publication}

Not applicable.

\section{Availability of data and materials}

All the data from electronic medical records in Tongji Hospital were reviewed by experienced physicians separately and checked by 2 physicians independently.

\section{Competing interests}


The authors declare that they have no competing interests.

\section{Funding}

This project was supported by project grant 81870013 from the National Natural Science Foundation of China; and grant 7202178 from the National Natural Science Foundation of Beijing.

\section{Authors' contributions}

Yanming Li, Xiaomao Xu and Xunliang Tong contributed to the conception and design of the study and interpretation of the results. Xunliang Tong and Xueting Yuan drafted the manuscript. Xunliang Tong, Xuefeng Zhong, He Wang and Wei Zhou contributed to the acquisition of the data and revision of the manuscript for important intellectual content. Anqi Cheng performed the statistical analysis and revised the manuscript for important intellectual content. All authors read and approved the final manuscript.

\section{Acknowledgements}

The authors thank Academician Chen Wang for his guidance and assistance with this work.

\section{References}

1. N. Zhu, D. Zhang, W. Wang, X. Li, B. Yang, J. Song, et al. A Novel Coronavirus from Patients with Pneumonia in China, 2019. N Engl J Med. 2020; 382: 727-733.

2. W.H. Organization. A public health emergency of international concern over the global outbreak of novel coronavirus declared by WHO. Available at: https://www.who.int/emergencies/diseases/novelcoronavirus-2019/events-as-they-happen

3. C. Huang, Y. Wang, X. Li, L. Ren, J. Zhao, Y. Hu, et al. Clinical features of patients infected with 2019 novel coronavirus in Wuhan, China. Lancet. 2020; 395: 497-506.

4. H. Li, L. Liu, D. Zhang, J. Xu, H. Dai, N. Tang, et al. SARS-CoV-2 and viral sepsis: observations and hypotheses. Lancet. 2020; 395: 1517-1520.

5. N. Vabret, G.J. Britton, C. Gruber, S. Hegde, J. Kim, M. Kuksin, et al. Immunology of COVID-19: Current State of the Science. Immunity. 2020; 52: 910-941.

6. M. Yang, C.K. Li, K. Li, K.L. Hon, M.H. Ng, P.K. Chan, et al. Hematological findings in SARS patients and possible mechanisms (review). Int J Mol Med. 2004; 14: 311-315.

7. H. Chu, J. Zhou, B.H. Wong, C. Li, J.F. Chan, Z.S. Cheng, et al. Middle East Respiratory Syndrome Coronavirus Efficiently Infects Human Primary T Lymphocytes and Activates the Extrinsic and Intrinsic Apoptosis Pathways. J Infect Dis. 2016; 213: 904-914.

8. R. Channappanavar, J. Zhao, S. Perlman. T cell-mediated immune response to respiratory coronaviruses. Immunol Res. 2014; 59: 118-128.

9. C. Qin, L. Zhou, Z. Hu, S. Zhang, S. Yang, Y. Tao, et al. Dysregulation of Immune Response in Patients with Coronavirus 2019 (COVID-19) in Wuhan, China. Clin Infect Dis. 2020; 71: 762-768. 
10. D. Li, Y. Chen, H. Liu, Y. Jia, F. Li, W. Wang, et al. Immune dysfunction leads to mortality and organ injury in patients with COVID-19 in China: insights from ERS-COVID-19 study. Signal Transduct Target Ther. 2020; 5: 62.

11. L. Tan, Q. Wang, D. Zhang, J. Ding, Q. Huang, Y.Q. Tang, et al. Lymphopenia predicts disease severity of COVID-19: a descriptive and predictive study. Signal Transduct Target Ther. 2020; 5: 33.

12. X. Yang, Y. Yu, J. Xu, H. Shu, J.a. Xia, H. Liu, et al. Clinical course and outcomes of critically ill patients with SARS-CoV-2 pneumonia in Wuhan, China: a single-centered, retrospective, observational study. Lancet Respir Med. 2020; 8: 475-481.

13. A. Chou, J.A. Serpa. Eosinophilia in patients infected with human immunodeficiency virus. Curr HIV/AIDS Rep. 2015; 12: 313-316.

14. J.M. Rodrigo-Munoz, B. Sastre, J.A. Canas, M. Gil-Martinez, N. Redondo, V. Del Pozo. Eosinophil Response Against Classical and Emerging Respiratory Viruses: COVID-19. J Investig Allergol Clin Immunol. 2020.

15. C.E. Lavoignet, P. Le Borgne, S. Chabrier, J. Bidoire, H. Slimani, J. Chevrolet-Lavoignet, et al. White blood cell count and eosinopenia as valuable tools for the diagnosis of bacterial infections in the ED. Eur J Clin Microbiol Infect Dis. 2019; 38: 1523-1532.

16. G. Xie, F. Ding, L. Han, D. Yin, H. Lu, M. Zhang. The role of peripheral blood eosinophil counts in COVID-19 patients. Allergy. 2020.

17. D.A. Bass. Behavior of eosinophil leukocytes in acute inflammation. II. Eosinophil dynamics during acute inflammation. J Clin Invest. 1975; 56: 870-879.

18. A. Allegra, M. Di Gioacchino, A. Tonacci, C. Musolino, S. Gangemi. Immunopathology of SARS-CoV-2 Infection: Immune Cells and Mediators, Prognostic Factors, and Immune-Therapeutic Implications. Int J Mol Sci. 2020; 21.

19. K. Li, Z. Hao, X. Zhao, J. Du, Y. Zhou. SARS-CoV-2 infection-induced immune responses: Friends or foes? Scand J Immunol. 2020; 92: e12895.

20. F. Zhang, R. Gan, Z. Zhen, X. Hu, X. Li, F. Zhou, et al. Adaptive immune responses to SARS-CoV-2 infection in severe versus mild individuals. Signal Transduct Target Ther. 2020; 5: 156.

21. L. Tan, Q. Wang, D. Zhang, J. Ding, Q. Huang, Y.Q. Tang, et al. Correction: Lymphopenia predicts disease severity of COVID-19: a descriptive and predictive study. Signal Transduct Target Ther. 2020; 5: 61 .

22. H.F. Rosenberg, S. Phipps, P.S. Foster Eosinophil trafficking in allergy and asthma. J Allergy Clin Immunol. 2007; 119: 1303-1310; quiz 1311-1302.

23. S.F. Pedersen, Y.C. Ho. SARS-CoV-2: a storm is raging. J Clin Invest. 2020; 130: 2202-2205.

24. J.L. Moran, P.L. Graham, S. Rockliff, A.D. Bersten. Updating the evidence for the role of corticosteroids in severe sepsis and septic shock: a Bayesian meta-analytic perspective. Crit Care. 2010; 14: R134. 
25. S. Jiang, T. Liu, Y. Hu, R. Li, X. Di, X. Jin, et al. Efficacy and safety of glucocorticoids in the treatment of severe community-acquired pneumonia: A meta-analysis. Medicine. 2019; 98: e16239.

26. P.C. Fulkerson, M.E. Rothenberg. Targeting eosinophils in allergy, inflammation and beyond. Nat Rev Drug Discov. 2013; 12: 117-129.

27. R.A. Siemieniuk, J.J. Bartoszko, L. Ge, D. Zeraatkar, A. Izcovich, H. Pardo-Hernandez, et al. Drug treatments for covid-19: living systematic review and network meta-analysis. BMJ. 2020; 370: $\mathrm{m} 2980$.

\section{Tables}

Table 1 Demographic and baseline characteristics of COVID-19 hospitalized patients 


\begin{tabular}{|c|c|c|c|c|}
\hline Characteristics & $\begin{array}{l}\text { All Patients } \\
(n=198)\end{array}$ & $\begin{array}{l}\text { Survivor } \\
(n=185)\end{array}$ & $\begin{array}{l}\text { Non-survivor } \\
(n=13)\end{array}$ & $\mathbf{P}$ \\
\hline \multicolumn{5}{|l|}{ Demographic } \\
\hline Age, median (IQR), yrs & $62.5(48,69)$ & $62(48,69)$ & $68(58,84)$ & 0.065 \\
\hline Gender, Male, n (\%) & $99(50 \%)$ & $90(48.6 \%)$ & $9(69.2 \%)$ & 0.251 \\
\hline Smoking, n (\%) & $6(3.1 \%)$ & $6(3.3 \%)$ & 0 & 1.000 \\
\hline \multicolumn{5}{|l|}{ Comorbidities, n (\%) } \\
\hline Chronic respiratory disease & $11(5.7 \%)$ & $11(6.1 \%)$ & 0 & 1.000 \\
\hline Malignancy & $7(3.6 \%)$ & $4(2.2 \%)$ & $3(23.1 \%)$ & 0.007 \\
\hline Hypertension & $76(40.0 \%)$ & 72(40.7\%) & $4(30.8 \%)$ & 0.568 \\
\hline Diabetes & $32(16.7 \%)$ & $30(16.8 \%)$ & $2(15.4 \%)$ & 1.000 \\
\hline Cardiovascular disease & $10(5.2 \%)$ & $9(5.0 \%)$ & $1(7.7 \%)$ & 0.513 \\
\hline Chronic kidney disease & $6(3.1 \%)$ & $6(3.4 \%)$ & 0 & 1.000 \\
\hline \multicolumn{5}{|l|}{$\begin{array}{l}\text { Signs and symptoms, } \\
\text { n (\%) }\end{array}$} \\
\hline Fever & $153(79.7 \%)$ & $142(79.3 \%)$ & $11(84.6 \%)$ & 1.000 \\
\hline Chills/shivers & $42(21.2 \%)$ & $40(21.6 \%)$ & $2(15.4 \%)$ & 0.739 \\
\hline Cough & $124(64.9 \%)$ & $117(65.7 \%)$ & $7(53.8 \%)$ & 0.386 \\
\hline Productive cough & $61(31.9 \%)$ & $57(32.0 \%)$ & $4(30.8 \%)$ & 1.000 \\
\hline Chest pain/chest congestion & $38(19.9 \%)$ & $35(19.7 \%)$ & $3(23.1 \%)$ & 0.725 \\
\hline Dyspnea & $66(34.6 \%)$ & $61(34.3 \%)$ & $5(38.5 \%)$ & 0.759 \\
\hline Diarrhea & $57(29.7 \%)$ & $54(30.2 \%)$ & $3(23.1 \%)$ & 0.758 \\
\hline Fatigue or myalgia & $83(41.9 \%)$ & $77(41.6 \%)$ & $6(46.2 \%)$ & 0.749 \\
\hline \multicolumn{5}{|l|}{ Oxygen Therapy, n (\%) } \\
\hline Nasal cannula & $87(43.9 \%)$ & $87(47.0 \%)$ & 0 & 0.000 \\
\hline Oxygen mask & $4(2.0 \%)$ & $3(1.6 \%)$ & $1(7.7 \%)$ & 0.257 \\
\hline NMV+ High-flow nasal cannula & $84(42.4 \%)$ & $79(42.7 \%)$ & $5(38.5 \%)$ & 0.576 \\
\hline IMV & $11(5.6 \%)$ & $4(2.2 \%)$ & $7(53.8 \%)$ & 0.000 \\
\hline ECMO & $2(1.0 \%)$ & $2(1.1 \%)$ & 0 & 1.000 \\
\hline
\end{tabular}




\begin{tabular}{|lllll|}
\hline Drugs, $\mathbf{n}(\%)$ & & & \\
\hline Oseltamivir & $60(36.4 \%)$ & $58(37.9 \%)$ & $2(16.7 \%)$ & 0.214 \\
\hline Arbidol & $121(71.2 \%)$ & $113(71.5 \%)$ & $8(66.7 \%)$ & 0.745 \\
\hline Lopinavir + Ritonavir & $23(14.2 \%)$ & $10(6.7 \%)$ & $13(100 \%)$ & 0.000 \\
\hline Ribavirin & $9(5.6 \%)$ & $8(5.4 \%)$ & $1(8.3 \%)$ & 0.672 \\
\hline Glucocorticoid & $42(25.8 \%)$ & $34(22.5 \%)$ & $8 \llbracket 66.7 \% \square$ & 0.002 \\
\hline CURB-65* & & & & 0.000 \\
\hline $0-1$ & $170(86.7 \%)$ & $170(92.9 \%)$ & 0 & 0.000 \\
\hline 2 & $14(7.1 \%)$ & $13(7.1 \%)$ & $1(7.7 \%)$ & 1.000 \\
\hline $3-5$ & $12(6.1 \%)$ & $0(0)$ & $12(92.3 \%)$ & 0.000 \\
\hline Hospital length of stay, days & $14(10,18)$ & $14(11,18)$ & $8(4,12)$ & 0.000 \\
\hline Time from illness onset to discharge, days & $27(22,34)$ & $27(23,34)$ & $20(9,28)$ & 0.005 \\
\hline
\end{tabular}

${ }^{*}$ Curb-65 score (confusion, uremia, the elevated respiratory rate, hypotension, and age $\geq 65$ ) stratify patients into three levels: low-risk ( $\leq 1)$, intermediate-risk (2) and high-risk ( $\geq 3)$.

Table 2 Initial laboratory findings among survivors and non-survivors 


\begin{tabular}{|c|c|c|c|c|}
\hline & $\begin{array}{l}\text { All Patients } \\
(n=198)\end{array}$ & $\begin{array}{l}\text { Survivor } \\
(n=185)\end{array}$ & $\begin{array}{l}\text { Non-survivor } \\
(n=13)\end{array}$ & $\mathbf{P}$ \\
\hline \multicolumn{5}{|l|}{ Hematologic } \\
\hline White blood cells, $\times 10^{9} / \mathrm{L}$ & $5.46(4.19,7.10)$ & $5.42(4.17,6.83)$ & $6.73(3.93,9.37)$ & 0.248 \\
\hline Neutrophils, $\times 10^{9} / \mathrm{L}$ & $3.43(2.43,4.81)$ & $3.36(2.40,4.66)$ & $5.58(2.99,8.41)$ & 0.024 \\
\hline Neutrophil percentage, $\%$ & $66.5(55.6,74.0)$ & $65.5(55.4,73.1)$ & $83.6(76.4,89.8)$ & 0.000 \\
\hline Lymphocytes, $\times 10^{9} / \mathrm{L}$ & $1.02(0.78,1.52)$ & $1.08(0.78,1.57)$ & $0.50(0.33,0.82)$ & 0.000 \\
\hline Lymphocyte percentage, $\%$ & $23.4(15.1,31.6)$ & $24.5(16.0,32.8)$ & $10.9(5.25,16.9)$ & 0.000 \\
\hline $\begin{array}{l}\text { Lymphocytes }<0.8 \times 10^{9} / \mathrm{L} \\
\mathrm{n}(\%)\end{array}$ & $75(37.9 \%)$ & $65(35.1 \%)$ & $10(76.9 \%)$ & 0.005 \\
\hline Neutrophil-to-lymphocyte ratio & $3.15(1.80,5.59)$ & $3.05(1.76,5.17)$ & $8.43(4.52,18.61)$ & 0.000 \\
\hline Monocytes, $\times 10^{9} / \mathrm{L}$ & $0.46(0.33,0.62)$ & $0.47(0.34,0.63)$ & $0.33(0.21,0.60)$ & 0.168 \\
\hline Monocyte percentage, \% & $8.5(6.5,10.3)$ & $8.7(6.9,10.4)$ & $5.9(3.5,8.2)$ & 0.000 \\
\hline Eosinophils, $\times 10^{9} / \mathrm{L}$ & $0.03(0.00,0.09)$ & $0.03(0.00,0.10)$ & $0.00(0.00,0.00)$ & 0.000 \\
\hline Eosinophil percentage, \% & $0.55(0.00,1.70)$ & $0.70(0.00,1.75)$ & $0.00(0.00,0.00)$ & 0.000 \\
\hline $\begin{array}{l}\text { Eosinophil }<0.02 \times 10^{9} / \mathrm{L} \\
\mathrm{n}(\%)\end{array}$ & $78(39.4 \%)$ & 65(35.1\%) & $13(100 \%)$ & 0.000 \\
\hline Basophils, $\times 10^{9} / \mathrm{L}$ & $0.01(0.01,0.02)$ & $0.01(0.01,0.02)$ & $0.00(0.00,0.01)$ & 0.001 \\
\hline Basophil percentage, \% & $0.2(0.1,0.4)$ & $0.2(0.1,0.4)$ & $0.00(0.00,0.01)$ & 0.000 \\
\hline Red blood cells, $\times 10^{12} / \mathrm{L}$ & $4.10(3.70,4.45)$ & $4.10(3.75,4.46)$ & $3.39(1.77,4.37)$ & 0.013 \\
\hline Platelets, $\times 10^{9} / \mathrm{L}$ & $225(163,301)$ & $232(171,305)$ & $145(76,241)$ & 0.007 \\
\hline PLT $<100 \times 10^{9} / \mathrm{L}, \mathrm{n}(\%)$ & $12(6.1 \%)$ & $8(4.3 \%)$ & $4(30.8 \%)$ & 0.004 \\
\hline Hemoglobin, g/L & $124(114,137)$ & $125(115,137)$ & $107(55,136)$ & 0.021 \\
\hline \multicolumn{5}{|l|}{ Biochemical test } \\
\hline Albumin, $\mathrm{g} / \mathrm{L}$ & $36(32,39)$ & $36.1(32.4,39.2)$ & $32.8(28.7,36.1)$ & 0.023 \\
\hline $\mathrm{ALT}, \mathrm{U} / \mathrm{L}$ & $22(15,39)$ & $23(15,40)$ & $19(17,33)$ & 0.482 \\
\hline AST, U/L & $27(19,38)$ & $27(19,37)$ & $47(36,58)$ & 0.005 \\
\hline Creatinine, $\mu \mathrm{mol} / \mathrm{L}$ & $69.5(58,86)$ & $69(58,83)$ & $99(61,114)$ & 0.019 \\
\hline
\end{tabular}




\begin{tabular}{|c|c|c|c|c|}
\hline $\mathrm{LDH}, \mathrm{U} / \mathrm{L}$ & $261(206,328)$ & 258(205,318) & $400(302,674)$ & 0.001 \\
\hline \multicolumn{5}{|l|}{ Other indices } \\
\hline $\begin{array}{l}\text { Erythrocyte sedimentation } \\
\text { rate, } \mathrm{mm} / \mathrm{h}\end{array}$ & $40(18.64)$ & $39(18,64)$ & $50(12,71)$ & 0.953 \\
\hline Serum ferritin, $\mathrm{ng} / \mathrm{mL}$ & $522(310,893)$ & $480(308,799)$ & $1968(1520,3507)$ & 0.001 \\
\hline $\mathrm{IL}-6, \mathrm{pg} / \mathrm{mL}$ & $11.61(4.21,27.12)$ & $9.33(3.96,21.14)$ & $41.6(23.2,65.3)$ & 0.003 \\
\hline C-reactive protein, $\mathrm{mg} / \mathrm{L}$ & $23.1(3.2,50.2)$ & $16.8(2.8,45.0)$ & $72.4(44.8,165.0)$ & 0.000 \\
\hline Troponin个, n (\%) & $24(18.0 \%)$ & $14(11.7 \%)$ & $10(76.9 \%)$ & 0.000 \\
\hline NT-proBNP, pg/mL & $157(64,411)$ & $127(62,335)$ & $554(483,995)$ & 0.000 \\
\hline PT, s & $13.8(13.3,14.3)$ & $13.7(13.2,14.2)$ & $14.9(13.9,16.0)$ & 0.002 \\
\hline APTT, s & $39.7(36.6,43.9)$ & $39.6(36.6,43.4)$ & $45.4(37.8,47.2)$ & 0.105 \\
\hline FIB, g/L & $4.71(3.73,5.85)$ & $4.65(3.75,5.81)$ & $4.87(2.61,6.11)$ & 0.763 \\
\hline D-Dimer, $\mu \mathrm{g} / \mathrm{mL}$ & $0.91(0.46,1.80)$ & $0.76(0.46,1.67)$ & $2.01(1.10,5.10)$ & 0.009 \\
\hline D-Dimer $\uparrow, n(\%)$ & 116(63.7\%) & $105(62.1 \%)$ & $11(84.6 \%)$ & 0.138 \\
\hline
\end{tabular}

Table 3 Clinical characteristics of patients according to eosinophils level on admission. 


\begin{tabular}{|c|c|c|c|}
\hline & $\begin{array}{l}\operatorname{EOS}\left(<0.02 \times 10^{9} / L\right) \\
(n=78)\end{array}$ & $\begin{array}{l}\operatorname{EOS}\left(\geq 0.02 \times 10^{9} / L\right) \\
(n=120)\end{array}$ & $\mathbf{P}$ \\
\hline Age, median (IQR), yrs & $64(53,71)$ & $61(47,69)$ & 0.126 \\
\hline Gender, Male, n (\%) & $41(52.6 \%)$ & $58(48.3)$ & 0.561 \\
\hline \multicolumn{4}{|l|}{ Signs and symptoms, n (\%) } \\
\hline Fever & 64(85.3\%) & $89(76.1 \%)$ & 0.120 \\
\hline Chills/shivers & $18(23.1 \%)$ & $24(20.0 \%)$ & 0.605 \\
\hline Cough & $46(61.3 \%)$ & $78(67.2 \%)$ & 0.403 \\
\hline Productive cough & $61(31.9 \%)$ & $22(29.3 \%)$ & 0.535 \\
\hline Chest pain/Chest congestion & $16(21.3 \%)$ & $22(19.0 \%)$ & 0.689 \\
\hline Dyspnea & $26(34.7 \%)$ & $40(34.5 \%)$ & 0.979 \\
\hline Diarrhea & $17(22.7 \%)$ & $40(34.2 \%)$ & 0.088 \\
\hline Fatigue or myalgia & $28(35.9 \%)$ & $55(45.8 \%)$ & 0.166 \\
\hline Highest temperature, ${ }^{\circ} \mathrm{C}$ & $38.9(38.4,39.0)$ & $38.5(38.0,39.0)$ & 0.012 \\
\hline CURB-65, n (\%) & & & 0.000 \\
\hline $0-1$ & $59(76.6 \%)$ & $111(93.3 \%)$ & 0.001 \\
\hline 2 & $6(7.8 \%)$ & $8(6.7 \%)$ & 0.783 \\
\hline $3-5$ & $12(15.6 \%)$ & $0(0 \%)$ & 0.000 \\
\hline Glucocorticoid, n (\%) & $30(44.8 \%)$ & $12(12.5 \%)$ & 0.000 \\
\hline Duration of viral shedding, days & $26(21,32)$ & $26(20,33)$ & 0.753 \\
\hline Hospital length of stay, days & $15(10,18)$ & $13(11,17)$ & 0.609 \\
\hline Time from illness onset to discharge, days & $25(20,32)$ & $28(23,35)$ & 0.046 \\
\hline Death, n (\%) & 13(16.7\%) & $0(0 \%)$ & 0.000 \\
\hline
\end{tabular}

\section{Figures}



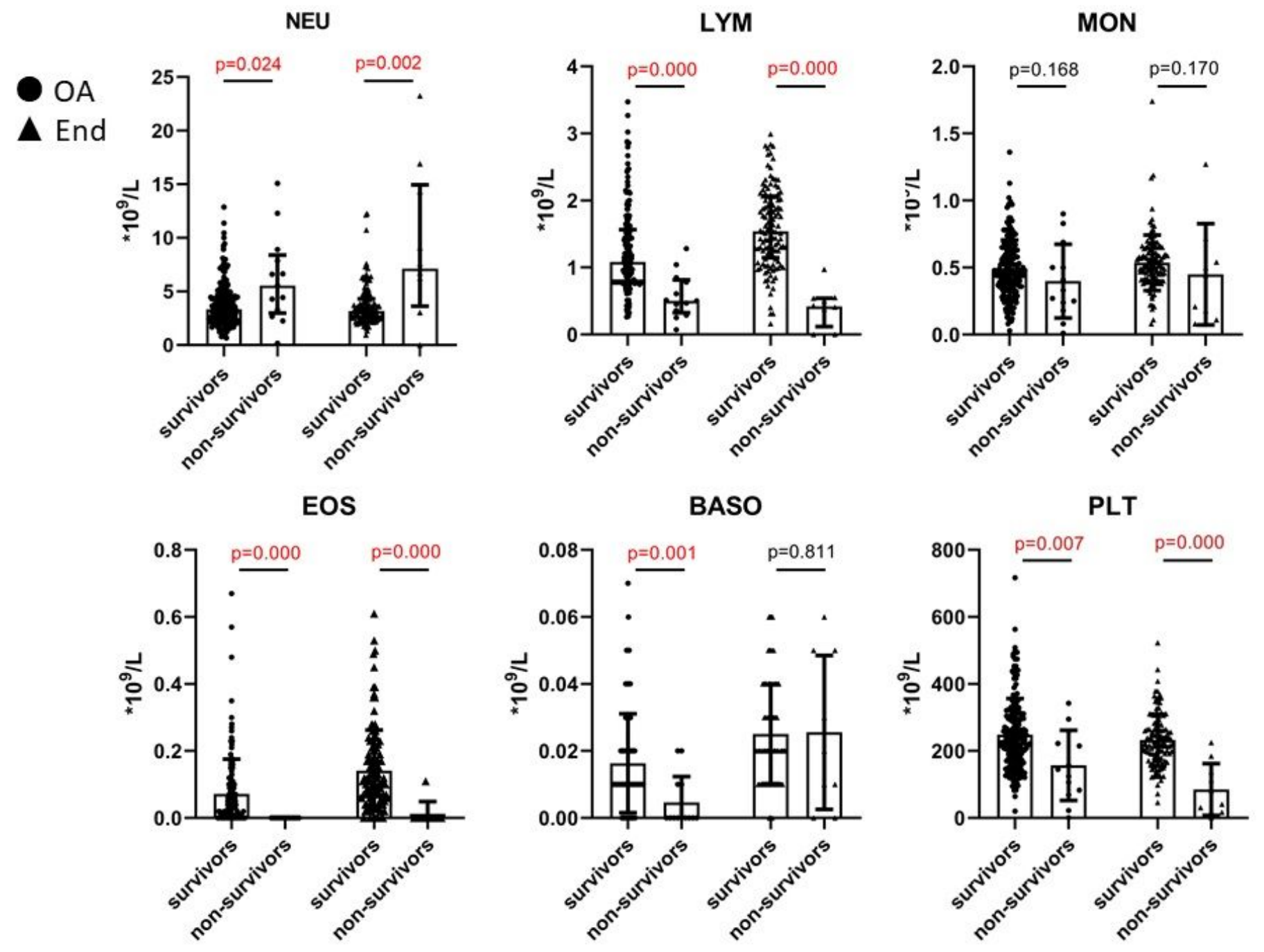

Figure 1

the prolonging of PT and increased levels of D-dimer were significantly higher in non-survivors compared to survivors 
A
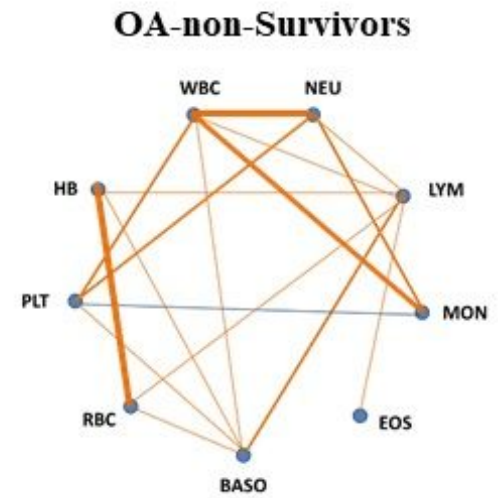

C

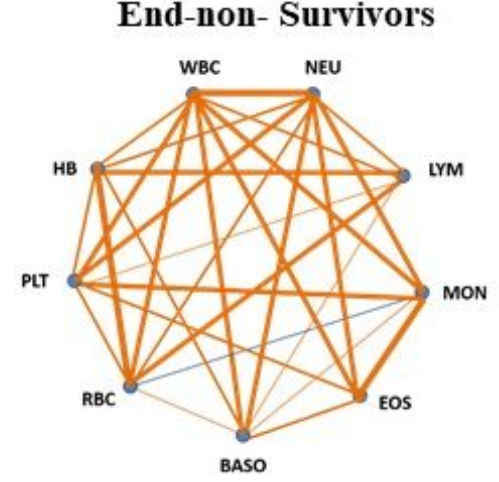

B

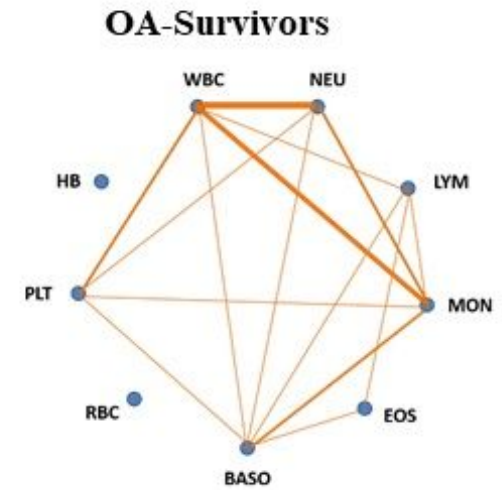

D

\section{End- Survivors}

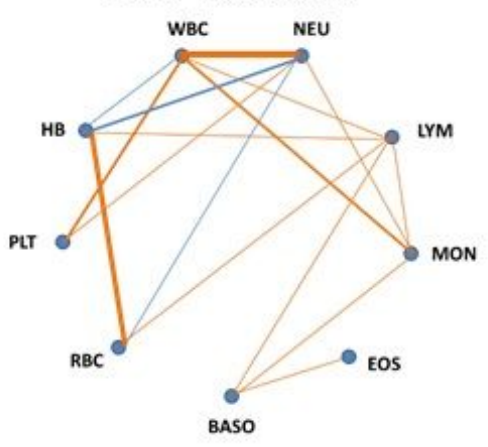

\section{Figure 2}

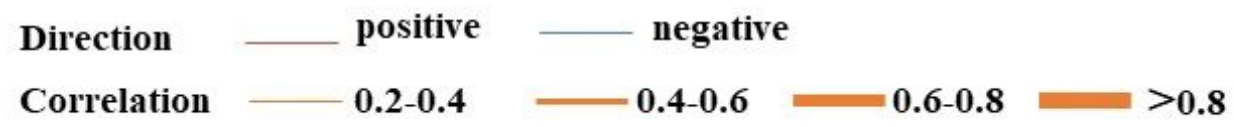

non-survivors showed similar positive strong correlations between the counts of EOS and BASO ( $r$ $=0.562$ ) (Figure 2). 
A
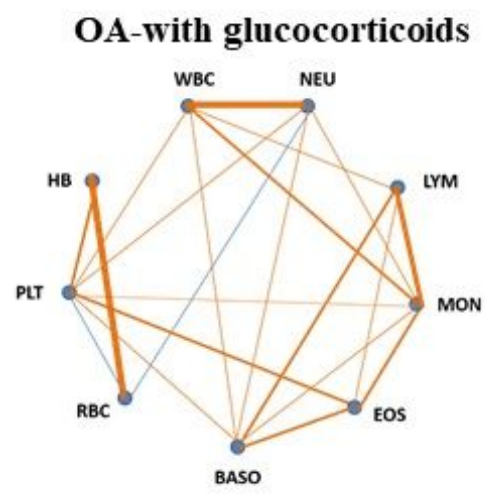

C

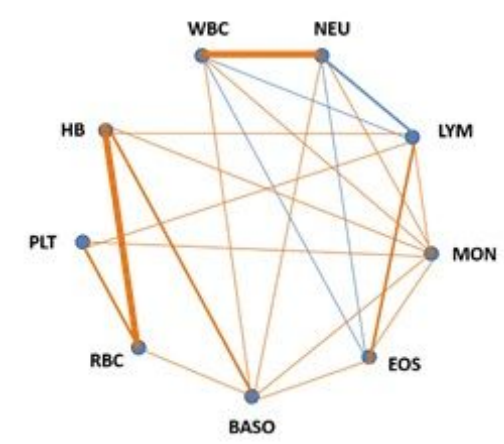

B

\section{OA-without glucocorticoids}

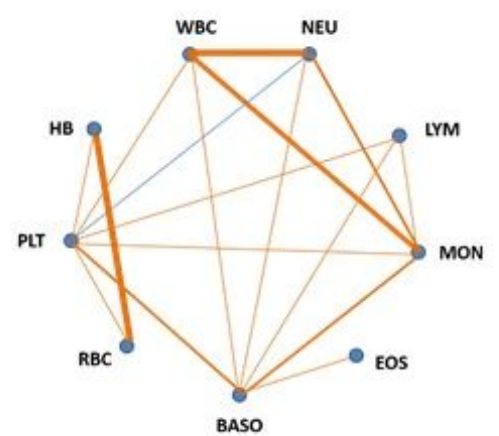

D

End- without glucocorticoids

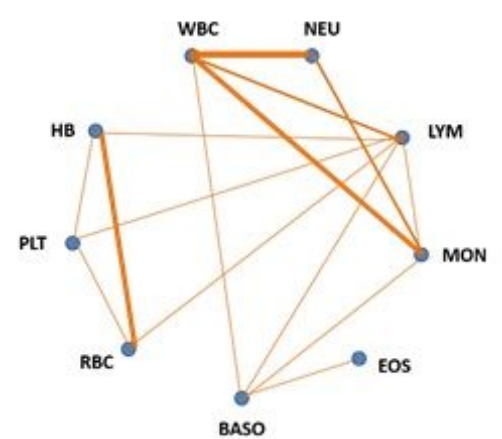

Direction positive

negative

Correlation

0.2-0.4

0.4-0.6

$0.6-0.8$

$>0.8$

\section{Figure 3}

A similar correlation was observed between the counts of LYM and EOS in patients received glucocorticoids therapy $(r=0.454)$, but no correlation was found before the treatment $(r=0.020)$ (Figure 3). 